\title{
Common Carotid Artery Dissection and Anastomosis Techniques on the Rat (End to End Anastomosis)
}

\author{
${ }^{1}$ Hilmi Özden, ${ }^{1}$ Hakan Ay, ${ }^{1}$ Gül Güven, ${ }^{2}$ Sibel Canbaz Kabayıç \\ ${ }^{1}$ Eskişehir Osmangazi University, Faculty of Medicine, Department of Anatomy \\ ${ }^{2}$ Dumlupınar Üniversitesi, University, Faculty of Medicine, Department of Neurology \\ *email: hilmiozden@gmail.com
}

\begin{abstract}
The authors in this article are to explain common carotid artery dissection and anastomosis microsurgical techniques on the rat.

KEYWORDS: Rat, Common Carotid Artery, Anastomosis.

\section{SIÇANDA ARTERIA CAROTIS COMMUNIS DİSEKSIYYON VE ANASTOMOZ TEKNIKLERİ (UÇ-UCA ANASTOMOZ)}

ÖZET: Bu makalede yazarlar, sıçanlarda arteria carotis communis diseksiyonu ve anastomozu ile ilgili mikrocerrahi teknikleri hakkında bilgiler verip, açıklamalar yapmaktadırlar.

ANAHTAR KELIMLER: Sıçan, arteria carotia communis, anastomoz.
\end{abstract}

\section{Introduction}

Although many surgeons use the operative microscope every day, the techniques for suturing very small vascular and neural structures are substantially more complex and unfamiliar than the routine. Mastery of microsurgical technique is both rewarding and transferable to other aspects of surgery. Practice is the key and requires dedication of time and effort (1).

\section{Basic Instruments}

1. Magnification and illumination are essential when working with delicate tissues and fine suture material. A good-quality microscope should be used for both practice and surgery. Familiarity with the operative microscope will minimize the struggle to achieve optimal visualization. The microscope should be positioned to provide a comfortable neutral neck position and a relaxed posture of the arms as they rest on the operative surface. These adjustments prevent fatigue and tension in the neck and arms, which can amplify a native tremor. The microscope should be fitted with 10 to 15 eyepieces and a 200 to $300 \mathrm{~mm}$ focal length objective lens. For most exercises, a maximum magnification of 25 should suffice. In our study, a Carl Zeizz OPMI 99 microscope was used for end to end anastomosis.

2. The tools necessary to perform the microvascular anastomosis are few in number but highly specialized in nature. It is best to reserve a special set of instruments that will not be used for routine surgery. This will ensure that they are in good shape and reliable when they are needed. It is important to select tools that are comfortable to hold and employ without excessive effort. Several factors must be considered when selecting your tools. First, the shape of the portion of the tool that is held by your fingertips affects your ability to 
manipulate the tool without losing control. The most common shapes are either flat (like a typical forceps) or rounded. Some instruments have a knurled pattern cut into the metal surface to increase friction and grip (2).Micro scissor, forceps, vessel clamp as tools and 9-0 or 10-0 monofilament nylon suture with needle need to be prepared for the microsurgical procedure (Figure 1.A).

The tension of spring-loaded instruments (scissors and needle appliers) is also an important consideration. If the tension is too weak, it will be difficult to secure the tool between your fingertips without closing it excessively or dropping it from your grip. If the tension is too strong, it will require excessive effort that, over repeated use, will prompt premature fatigue.

The weight of the instrument also determines its usability. Instruments are typically made of either stainless steel or titanium. Stainless steel is heavier than titanium and, to some, has a more substantial feel between the fingertips. Finally, the length of the instrument handle determines comfortable working distance. Regardless of the depth of the tissue, it is critical that you be able to stabilize your hands by touching your small fingers to the working surface. During training exercises, it is easy to adjust the distance from the anastomosis because you will be working primarily on a flat surface. Fortunately, the most common anatomical location for microvascular anastomosis (the superficial temporal artery to middle cerebral artery) lies on the brain surface and the surrounding skull edge creates a nearly planar surface on which to stabilize one's hands (1).

\section{End-To-End Anastomosis}

End to end artery anastomosis in the living animal is one of the most important stages in microsurgery education. The common carotid artery and the femoral artery are the frequently used arteries. In microsurgery education the femoral artery is used more than the common carotid artery, which is likely used for investigational purposes (3). The femoral vessels, having bigger diameters, are easier to access and far from important structures. The femoral artery $(0.6-1 \mathrm{~mm}$ diameter), vein (1-2 mm diameter) and nerve reach to the leg through the femoral canal by passing beneath the middle part of the inguinal ligament. The anatomical location of this artery is more comfortable for the surgeon. Despite of these advantages of this artery, the flow of the femoral artery is lesser than the common carotid artery. The femoral artery has also a thinner media layer, and the probability of spasm is higher than the common carotid artery. In the light of these reasons, the risk for thromboses due to technical faults in the femoral artery is higher than the common carotid artery. The carotid arteries are one of the main arteries that supply the brain. But unlike the human, the vertebral arteries are sufficient to supply the brain with enough blood in rats when these arteries are ligated. This advantage gives us the opportunity to use these arteries in anastomosis studies. But the dissection of the carotid artery needs a lot of care due to the neighborhood of important structures like trachea, phrenic and vagal nerves. The carotid arteries are located deeper than the femoral arteries. A careful dissection must be made to have a clean sight for surgical procedures. It must be kept in mind that we can cause cervical luxation or obstruction of the trachea during the procedure, if we stretch the neck too much. Despite of these risks the common carotid artery gives no branches at the neck. Due to the thicker media layer the carotid artery is more appropriate for inexperienced surgeons and the spasms are not such a problem like in the femoral artery. That's why the carotid artery is a suitable model for inexperienced surgeons and increases the success chance of the surgical procedure that leads to an augmentation in the selfconfidence of the new surgeon $(3,4)$.

\section{Pharmacologic agents in Rats microsurgical procedures}

Sodium Pentobarbital (Nembutal $\left.{ }^{\circledR}\right)$ IP and Ketamin 12-15 $\mathrm{mg} / \mathrm{kg}$ im for anesthesia, Doxapram (Dopram $\left.{ }^{\circledR}\right)$ is a direct impulsory for central respiratory centre. IV Anticoagulant: Heparin, Vasodilator agents: Lidocaine (Xylocaine $\left.{ }^{\circledR}\right), \quad$ Rompun ${ }^{\circledR} \quad$ 5-7 $\mathrm{mg} / \mathrm{kg} \mathrm{IM}$ for sedation.

\section{Common Carotid Artery Dissection}

The carotid arteries lie under the sternomastoid and omohyoid muscles, over the scalene muscles and along the two sides of the trachea. A median skin incision has to be made in front of the neck. To reach the 
sternohyoid muscles, the overlapping superficial fat tissue has to be removed like a flap that has a superior peduncle. After then, the underlying muscles will be revealed. The sternohyoid muscles and lower located the sternomastoid, muscles that are coming from each site; merge on the midline like a "V" letter. The skin and underlying tissue over the sternomastoid muscle on the site that are chosen for dissection have to abrade laterally. The jugular vein will be seen in the fatty tissue below. After the sternomastoid muscle is revealed the surgeon has to give a reposition to the animal so the lateral side of the neck is fully in sight. To provide a comfortable workspace the sternomastoid muscle has to be removed by cutting at its lower and upper end. By this way the ecartation during the carotids dissection will be easier. After the sternomastoid muscle is removed the carotid artery will come in sight caudally. Cranially it is covered by the omohyoid muscle and that overlapping part has also be cuted and removed. A long carotid segment will be revealed with the accompanying vagal nerve. This nerve has to be separated from the artery under the dissection microscope with blunt dissection. After this step, a vessel clamp must be carried to the surgery field (carotid field) by using a forceps. The clamp sides should be moved to the widest point as possible. The clamp sides must be opened to hold the blood vessel and it is necessary to place a blue background sheet under the artery before it will be cut. Some important points must be considered before clamping the artery. First a background sheet must be placed. This procedure can cause spasms in the vascular structure. If this happens, or to prevent this, the operation area has to washed with warm isotonic salt solution. After dry up with a tampon the artery must irrigated with $\% 2$ lidocain. Then, the artery will dilate and relax in a few minutes. The clamping procedure requires great care. The clamps of the vessel clamp have to be diverged maximally so we have enough space to work between the clamps. It should be kept in mind that the vessel clamp must be tumbled during suturing and enough space is needed to make this maneuver possible. Another important point is to use the distal ends of the clamps to interrupt the blood flow (Figure 1.B). The proximal parts of the clamps may be wider located from each other so that maybe it can't make enough pressure to cease the blood flow. Moreover, the cut ends of the arteries can escape from the clamps because of the retraction after cutting. During clamping, firstly the clamp that is distal to the blood flow direction must be located, secondly the proximal clamp. An artery retracts about $25-30 \%$ after cutting. Therefore the clamps have to be moved to each other until the artery between slacks, before the cutting procedure. Another point is that the artery must be straight and not twisted. It can cause turbulences in the blood flow after anastomosis. After clamping, the rail of the vessel clamp must be kept in a horizontal position. It is an easy way to do that with underlying the proximal parts of the vessel clamp to the cut tissue (3).

\section{Artery Cut-off}

After this procedure the artery can be cut. The microscope must be used with a higher magnification. The best point for the anastomosis is the midpoint between the clamps. The micro scissor has to be positioned with a 90-degree angle to the blood vessel and the vessel must be cut with a single move. The vessel lumen should be irrigated with heparinized saline. By sliding the clip sides toward the midline of the clip rail, the cut ends of the vessel will come together (Figure 1.C). It is important that the cut edges are smooth and not squashed. It is advisable that the cut edges are observed under higher magnifications. So it will be controlled that the intimae are intact and still attached to the media. No thromboses and tissue remnants must be in and over the area that will be used for anastomosis. After then, a lower magnification can be used to wash the area. All blood and thromboses inside the vessel have to be washed out. A clean arterial lumen will appear white and transparent. These steps are very important for the success of this operation. This cleaning procedure can be carried out easily with a 5 cc injector filled with isotonic saline. If there are still blood remnants inside the vessel, a forceps or powerful irrigation can be used to take them out. After this step the arterial edges must be clean. It will be seen that the lumen isn't as wide as we think. A forceps, preferably a dilatation forceps, can be used to widen the lumen. To do that, we can hold the vessel with his adventitia with our passive hand and put the forceps with our active hand in the lumen 
with closed mouth. When we open the forceps inside the vessel it will dilate the vessel spontaneously. This procedure will also prevent spasms after the anastomosis.

Before proceeding the distance between the cut edges must be checked. When the cut edges are raised, with forceps by holding them from their adventitia, the cut edges must be come together without be stretched, or the distance must not be greater than $1 \mathrm{~mm}$. Then the distance will be quiet right (Figure 1.D). If the distance between the cut edges is too much, the sutures will lacerate the vessel wall. In this case the clamps have to be adducted to each other with the use of a clamp forceps. There must be a small distance between the cut edges; but there must be no contact. If the dissection is well established, only a few adventitias will be pitched by the ends of the vessel. These parts should be hold and cut-off carefully. Another method is to capture adventitia by forceps and then to retract the rest of the adventitia on top of vessels.

After the procedures above the cut edges will be ready for suturing. But before, last controls have to be made:

- There must be no tissue remnants or blood clots in the sight of the microscope.

- The cut edges must be inspected a last time with a bigger magnification to be sure that there are no remnants in the vessel lumen.

- The distance between the cut edges must be under $1 \mathrm{~mm}$ or not greater than the lumen diameter.

- The clamps must be placed right and tight.

- The anesthesia of the animal must be controlled. If the respiration speed and the muscle tonus of the animal increase, a 1/5 doze of the given anesthetic must be administered (3).

\section{Anastomosis}

The aim of this step is to reestablish the continuity of the vessel lumen. In other words, the tube anatomy must be reestablished with a minimum of thrombogenic factors and irregularity that may disturbs the laminar flow. There are many suture techniques for this but single sutures are still the safest and most useful technique. Six to eight interrupted stitches with a 10/0 thread must be enough for the carotid artery (Figure 1.E). The first two sutures will be the hardest sutures. For this procedure the vessel contour must be imagined as a circle and the first suture named as the " 0 o suture". The other sutures will be named according their positions on this imaginary circle. It can be very difficult to control the cut edges and to determine the place of the first suture. The easiest way is to place the first two sutures with an 180 o angle to each other. After that two other sutures must be placed on the back and the fore side of the vessel (90o and 270o). The last step will be to place one suture between these first sutures $(3,4)$. One of the frequent faults seen during the first suture is sewing the front wall of the vessel to the back wall. If this fault is done in the last suture, this error can be only noticed after the clamps are putted up. Placing the sutures with different angles can reduce this risk. Suspending sutures can be placed to 0o-120o-240o. Or after placing two suspending sutures to $0 \mathrm{o}$ and $120 \mathrm{o}$, the front wall will be split up from the back wall under slight traction. Two tools are primarily used during anastomosis. The first one is the forceps in the passive hand and the needle holder with the needle in the dominant hand. The needle holder is used directly in holding and orienting of the thread during knotting. And the needle is used to help the forceps by holding tissues or to assist the forceps during maneuvers. The magnification of the dissection microscope has to be at lower to higher during suturing. The needle has to pierce first through the cut edge on the side of the dominant hand $(3,4)$. First the adventitia will be held with the pinpoint and the edge of the vessel is turned upwards. So the vessel lumen will be visible and we can insert the closed forceps tip and let it open inside the cut edge. With this maneuver the vessel lumen will be opened and we will be ready to meet the needle that passes the vessel wall. When the lumen is in our sight, it will be easy to see the passing needle and to hold it in the vessel lumen. Whereas the passive hand is in its position the needle penetrates in a right angle and must be moved with the gradient of the needle slightly through the vessel wall. After the needle is in the lumen it will be fallen between the tips of the forceps. When the needle holder reaches to the other side, the needle holder has to be released and the movement will be accomplished with the forceps. After the needle is pulled to the other side the needle holder have to be used again. 
Taking out the needle with a circular move will produce minimal trauma on the vessel wall. These traumas can be dangerous in rats. Holding the forceps tip open practically guides the needle tip that comes through the vessel wall inside the lumen. Furthermore, it prevents the needle from penetrating the back wall. It also supports the vessel wall while the needle pierces in the wall and facilitates to control the vessel. Holding the needle with the forceps needs attention or the delicate tip of the needle can become blunt. The forceps hold the needle first after the big part of it passes the wall. The thread is pulled a few centimeters after the needle is out of the vessel lumen. Then, the other cut edge must be pierced with the needle. The needle will be controlled again through holding it stable with the needle holder. Now the passive hand will pinch the adventitia of the cut edge on this side and elevate it until its lumen come in sight. At this time, the needle will be pierced to the inside through the wall and pulled out from outside. The first suture must be prepared through equal and symmetric parts of the cut edges. Otherwise the vessel will be twisted and this may affect the blood flow. And this may have the result that the surgical attempt will be unsuccessful. The penetrating points on each cut edges must have the same distance to the edges or the cut vessel walls will overlap during suturing. The needle must be pierced in this wall with a right angle too. The forceps in the passive hand will now support the vessel wall from outside. After the needle is put out, the thread has to draw out until the end of it is enough to make a ligature. Before pulling the thread it will be advisable to use a smaller magnification; so the needle holder can be seen during this process. Now the suture can be finished with 3 half ligatures. If the cut edges stretch the first half ligature too much so that it can't hold them together, the clamps of the vessel clamp must get a little bit closer. One of the two ends of the first ligature has to be longer than the other one. This long piece can be attached in to a notch on the background surface. This will help to control the vessel easier during the second ligature. The second ligature will be easier than the first one. Since the cut edges are even in touch. This ligature must be placed on 180 o relatively to the first one. The steps are the same like the first ligature. Letting here also a long end would help to stabilize the vessel (3).
By the first two ligatures the needle penetrates two times first in the first one and second in the other cut edge. But the following procedures are not like those because the cut edges are now tight in touch. By making the 90 o and 270 o ligatures the passive forceps can still be used to support the needle during piercing the vessel wall. But supporting the vessel wall from inside, during the following ligatures it is not possible. Stretching the vessel through its adventitia or the former ligatures will support the piercing process. After the ligatures 90o-45o-135o is placed on the front, the vessel clamp must be flipped, so ligatures can be placed on the backside (Figure 1.F) (3).

Before the last ligature it is advised to wash the lumen for a last time with isotonic saline. This procedure will clean the lumen and the vessel wall swells. This will show whether the ligatures are strong and the back wall is sutured to the front wall or not. If the vessel does not swell, it will indicate that something has gone wrong. In this instance every ligature have to be controlled by pulling it. The wrong ligatures must be removed and renewed (Figure 1.G).

At the end of suturing all ligatures must be controlled for errors that can cause leakage. After then the clamps can be opened and the vessel clamp removed. Lower magnification should be used for this procedure. First the clamp on the distal side of the blood flow has to be opened. Because of the lower blood pressure in this part it easier to control any possible leak. After the first clamp is removed small leakages can be observed. But they will stop shortly. After the environment is washed with isotonic saline and the fluid is drained, the other clamp can be opened (Figure 1.H). When the anastomosis is successful, the small leakages will stop in 1-2 minutes. During this waiting period absorbable haemostatic gauze can be put on the vessel $(3,4)$.

Finally, factors that affect the Anastomosis Procedure should mentioned; technical factors (faulty connecting of vessel walls, traumatized vessel walls due to forceps, faulty position of vessel walls, faulty traction suture strain, extra anastomotic strain, extra vessel clamp pressure, vessel torsion), flow factors (turbulence, stasis), coagulation factors (contamination, antithrombotic, prostaglandins), spasm. 

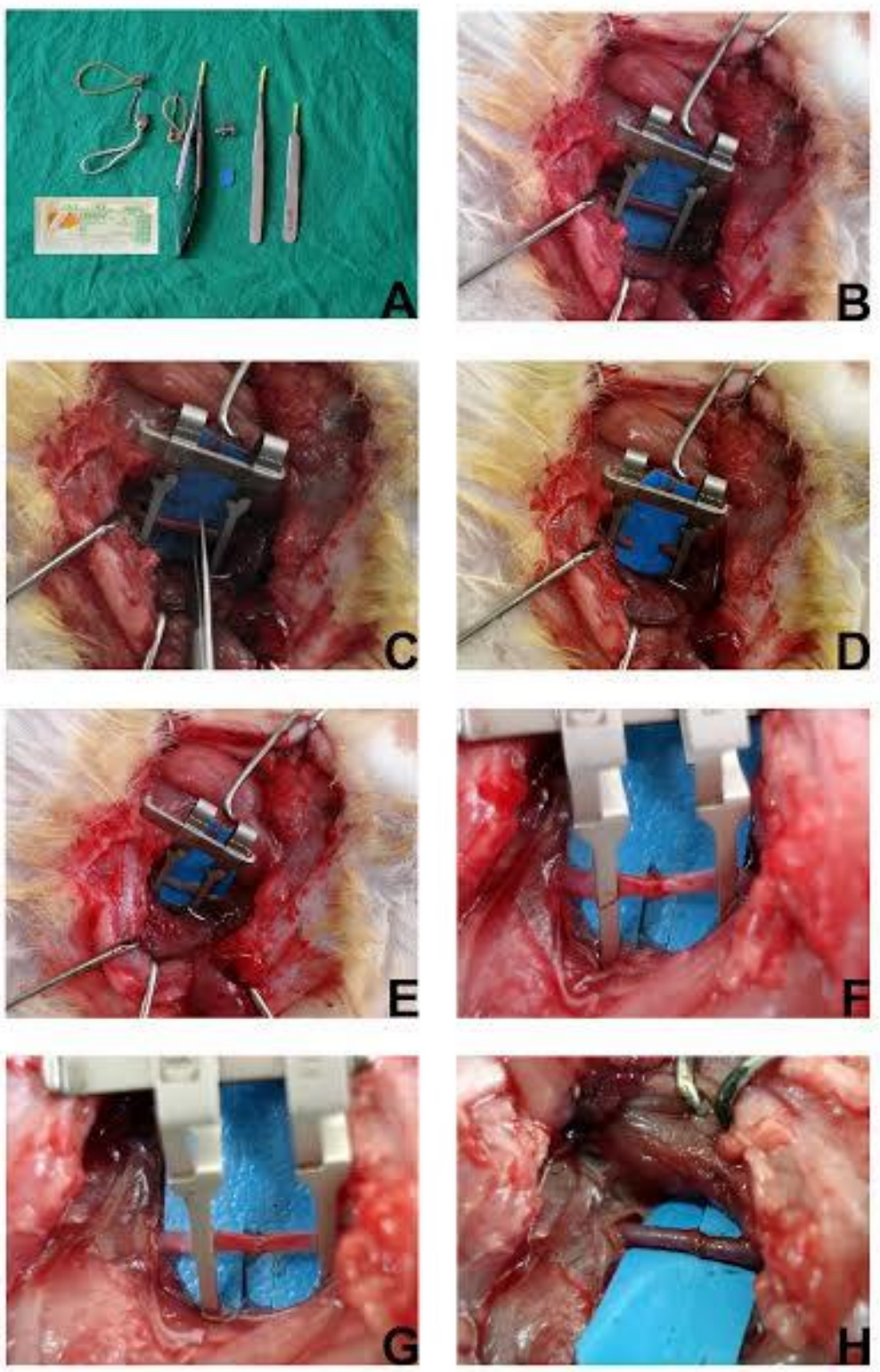

Figure 1: The equipment used in microsurgery and steps of the common carotid artery anastomosis. A. Tools (forceps, micro scissor, thread, vessel clamp, ecarteur) and 10/0 thread used in anastomosis B. Common carotid artery that is separated from the vagal nerve with a vessel clamp placed on it. $C$. Cutting of the clamped artery by a microscissor. D. Retraction of a clamped artery after cutting. E. Sutures on the front side of a clamped artery. F. Sutures on the backside of a clamped artery. G. End of the suturing procedure of a clamped artery. H. Reestablished blood flow after opening the vessel clamp.

\section{REFERENCES}

1. MacDonald, J. D. (2005). Learning to perform microvascular anastomosis. Skull Base, 15(3), 229.

2. Yasargil, M. G. (1969). Microsurgery applied to neurosurgery. G. Stuttgart: Georg Thieme Verlag, 119.
3. Bayramiçli, M. (2005). Deneysel Mikrocerrahi. Argos iletişim.1. Baskı, pp: 261-287.

4. Waynforth, H.B., Flecknell, P.A. (1992). Experimental and Surgical Technique in the Rat (second ed.), Academic, London. 